\title{
Word Processors with Line-Wrap: Cascading, Self-Organized Criticality, Random Walks, Diffusion, Predictability
}

\author{
Wolfgang Bauer and Scott Pratt \\ Department of Physics and Astronomy and National Superconducting Cyclotron Laboratory, Michigan State University, East \\ Lansing, MI 48824-1321, USA
}

(June 28, 1995)

\begin{abstract}
We examine the line-wrap feature of text processors and show that adding characters to previously formatted lines leads to the cascading of words to subsequent lines and forms a state of self-organized criticality. We show the connection to one-dimensional random walks and diffusion problems, and we examine the predictability of catastrophic cascades.
\end{abstract}

05.40.+j, 07.05.Tp, 89.80.+h, 91.30.Px

Composite systems may evolve to a critical state in which minor events may trigger a chain reaction that can effect an arbitrarily large number of constituents of the system. This state was called self-organized criticality [1] 3, and was first investigated for sandpiles. Theoretically and experimentally [4], avalanches in sandpiles show powerlaw distributions characteristic of real earthquakes [5.6]. The absence of an intrinsic length scale is attributed to self-organized criticality, where avalanches of all sizes contribute to keep the system perpetually in a critical state.

So far, no analytic solution to the model of Ref. [1] has been presented. However, if one introduces a preferred direction, then an exact analytic solution is possible [7.8], and a connection to the problem of two annihilating random walkers can be established.

Of particular interest is the question of predictability of catastrophic avalanches. In a recent experiment measuring the total mass of the sandpile it was found that a running total of small avalanches can predict the occurrence of large avalanches [9].

Here we introduce what we believe to be the most simple (and most directly connected to everyday experiences) example of self-organized criticality. Consider a modern word processor with line-wrap feature and fixed maximum number of characters per line. Such a word processor formats a paragraph without the explicit need to enter carriage returns or line feed characters. If a word is too long for a line, it is automatically wrapped into the next line. We consider an infinitely long paragraph formatted by this word processor. If one then adds another character to the beginning of the first line, the last word of this line may be wrapped to the second line, and a cascade of line-wraps may ensue. If a steady stream of characters or words is entered at the beginning of the first line, a sequence of cascades on all scales results, and the line lengths (excluding trailing blanks) in the paragraph form a self-organized critical state.

Let us - for the moment - neglect temporal correlations between the individual words shifted through the paragraph. (This assumes in practice that individual lines are infinitely long - an assumption we will relax again below.) In addition, we assume here for definiteness that the individual word lengths including one trailing blank are evenly distributed between 2 and $2\left\langle\ell_{w}\right\rangle-2$, where $\left\langle\ell_{w}\right\rangle$ is the average word length. (The probability for any given word length between these two limiting values is then $\left(2\left\langle\ell_{w}\right\rangle-3\right)^{-1}$.) For a fixed number of characters per line, the probability to have $b_{n}$ trailing blanks in this line can be shown to be

$$
p\left(b_{n}\right)= \begin{cases}\left\langle\ell_{w}\right\rangle^{-1} & \text { for } n=0,1 \\ \frac{2\left\langle\ell_{w}\right\rangle-2-b_{n}}{2\left\langle\ell_{w}\right\rangle^{2}-3\left\langle\ell_{w}\right\rangle} & \text { for } n \leq 1\end{cases}
$$

and consequently the average number of trailing blanks in a line is

$$
\left\langle b_{n}\right\rangle=\sum_{b_{n}=0}^{2\left\langle\ell_{w}\right\rangle-2} b_{n} p\left(b_{n}\right)=\frac{2}{3}\left\langle\ell_{w}\right\rangle-1+\frac{1}{3}\left\langle\ell_{w}\right\rangle^{-1} .
$$

In Fig. 1 (a) we display (crosses) the size distribution (= number of lines affected) for cascades in this system, where the total number of lines was chosen to be $10^{3}$, and $\left\langle\ell_{w}\right\rangle=6$. A total of $3 \times 10^{6}$ words $\left(=1.8 \times 10^{7}\right.$ characters $)$ were generated for this plot. One can clearly observe that for large number of lines, $n$, the distribution approaches the power law limit (solid line),

$$
N(n) \propto n^{-3 / 2} .
$$


It may be of interest that some experimental manifestations of self-organized criticality, such as the frequency distribution for power spectra in superconducting vortex avalanches [10], show similar power laws.

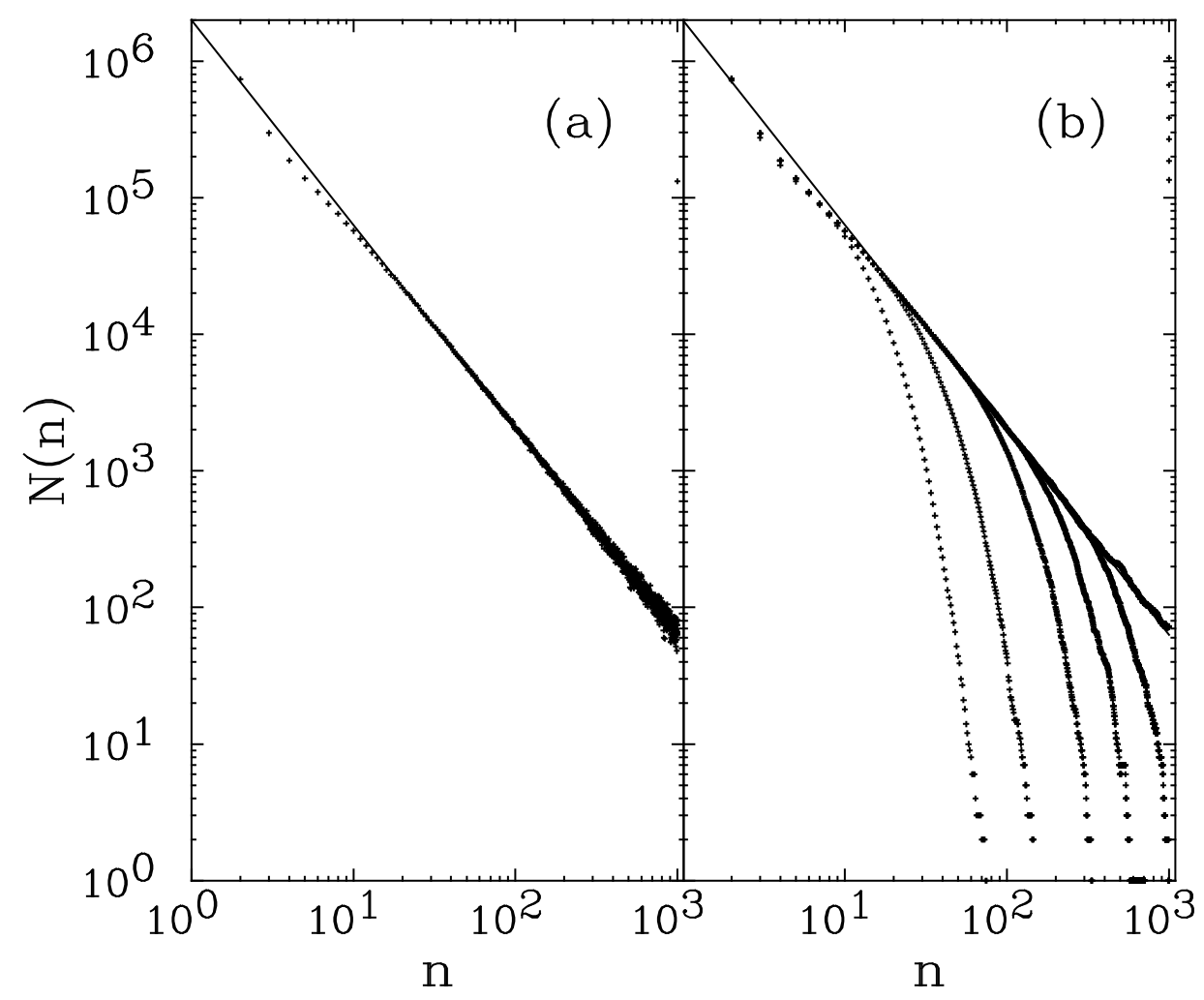

FIG. 1. Cascade lengths distribution for the word processor with line-wrap feature. (a) infinite line length, (b) finite line lengths, $\ell_{l}=20,30,50,70,100,200$, from left to right, respectively. The straight lines correspond to the asymptotic $n^{-3 / 2}$ solutions.

It is also of interest to compute the total activity, i.e. the total number of characters moved to different lines. The number distribution for this activity also approaches a power law, with an exponent of approximately $-4 / 3$ very much reminiscent of the earthquake strength distribution found in ref. [5].

We should point out here that none of our results depend on the type of the word length distribution chosen. We obtained for practical purposes identical results with Poissonian word length distributions.

The result of Eq. (3) can be understood by formulating the problem in terms of a random walk. One step in this random walk is the change in the number of blanks in a given line caused by a cascade passing through. To derive the step size distribution, we realize that pushing a word of length $\ell$ from line $n$ to $n+1$ increases the number of trailing blanks in line $n$ by $\ell$. Conversely, pushing a different word of length $\ell^{\prime}$ from line $n-1$ into line $n$ decreases the number of trailing blanks in line $n$ by $\ell^{\prime}$. The probability distribution for a change $\Delta b_{n}$ in the number of trailing blanks in line $n$ is then

$$
P\left(\Delta b_{n}\right)=\sum_{b_{n}=0}^{\infty} p\left(b_{n}\right) p\left(b_{n}-\Delta b_{n}\right)
$$

where $p\left(b_{n}\right)$ is the probability distribution for trailing blanks as given by Eq. (11). The probability distribution $P\left(\Delta b_{n}\right)$ is symmetric about $\Delta b_{n}=0$ and approximately triangular in shape. Thus its mean is 0 , and its variance is finite.

The total number of characters moved through line $n$ by the cascade is

$$
c_{n}=-\sum_{i=1}^{n-1} \Delta b_{i}
$$


If for any $n$, we have $c_{n} \leq b_{n}$, then the cascade terminates. Thus we see that our cascading problem is equivalent to a random walk problem with step size distribution given by Eq. (4). The result of Eq. (3) is the solution of the return-to-the-origin problem for a one-dimensional random walk.

It is, perhaps, more instructive to consider the corresponding continuum diffusion problem. The diffusion equation is

$$
\partial_{t} f(x, t)=D \partial_{x}^{2} f(x, t)
$$

with the boundary condition $f(0, t)=0$ and the solution

$$
f(x, t)=\frac{x}{4 \sqrt{\pi D^{3} t^{3}}} \exp \left[-x^{2} / 4 D t\right],
$$

where $t$ corresponds to the number of lines, $n$, in the random walk, and $x$ is the distance of the random walk to 0 . $D$ is the diffusion constant and can be calculated from the second moment of the random walk step size distribution, Eq. (化),

$$
D=\left\langle\Delta b_{n}^{2}\right\rangle / 2 .
$$

( $D \approx 6.3$ for the parameters used to produce Fig. 1. ) For the current at the origin we obtain

$$
J(t)=\left.D \partial_{x} f(x, t)\right|_{x=0} \propto t^{-3 / 2},
$$

in agreement with the numerical finding of Fig. 11.

We now proceed to study the case where we include all of the temporal correlations entailed by pushing an ordered (but individually randomly selected) sequence of words through our word processor. This is the case for finite line lengths. Inserting a number of characters equal to the line length, $\ell_{l}$, will result in a completely new first line, pushing the old first line into the second, and so on. Thus we get catastrophic cascades (= cascades involving all lines - 1000 in the specific example considered here) at least every $\ell_{l}$ characters.

In Fig. 1(b) we show our results using identical parameters to Fig. 11(a), but using finite line lengths, $\ell_{l}=20,30$, $50,70,100$, and 200. It can clearly be seen that the power law distribution is now cut off by an exponential depending on the line length. This behavior can be understood in terms of the random walk formulation of the problem. The finite line length corresponds to an additional absorbing barrier for the random walk, restricting $c_{n} \leq \ell_{l} \forall n$. The corresponding solution to the diffusion equation is

$$
f_{f}(x, t)=\sum_{j=1}^{\infty} C_{j} \sin \left(k_{j} x\right) \exp \left(-D k_{j}^{2} t\right)
$$

with long-time behavior dominated by $k_{1}$, where $k_{j}=\pi j / \ell_{l}$.

Of particular interest in studying models with 'random' catastrophic events is to investigate the limits of predictability of these events. To do this we record the number of characters, $\Delta c$, entered between catastrophic cascades. Using the same parameters as for the calculations in Fig. 1, we display in Fig. 2 (histogram) the number of events as a function of $\Delta c, N(\Delta c)$. It is obvious from this figure that they follow a Wigner-distribution,

$$
N(\Delta c) \propto(\Delta c /\langle\Delta c\rangle) \exp \left(-\pi(\Delta c /\langle\Delta c\rangle)^{2} / 4\right) .
$$

(As a side note we mention here that we obtain essentially identical behavior for finite line lengths, $\ell_{l}$, as long as $\ell_{l}$ is large compared to the mean value of the Wigner distribution, $\langle\Delta c\rangle$.) Also displayed in this figure (circles) is the distribution of the number of characters pushed into line $1000, c_{1000}$. This clearly follows the same functional form. From our above considerations of the diffusion equation we see that $N\left(c_{1000}\right) \rightarrow f(x, t=1000)$, and that therefore the mean number of characters entered between catastrophic cascades is

$$
\langle\Delta c\rangle=\sqrt{\pi D n}
$$

We thus see that both distributions displayed in Fig. 2 are governed by diffusion physics. 


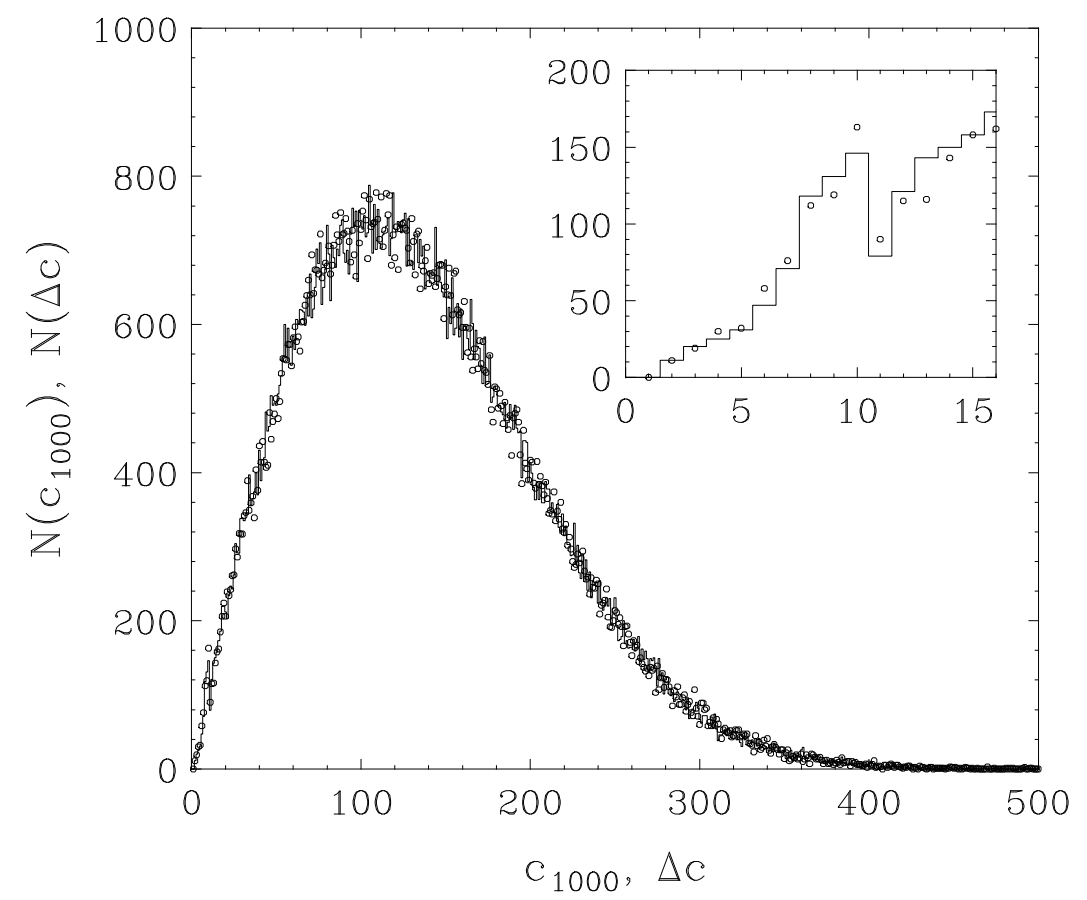

FIG. 2. Histogram: Number distribution, $N(\Delta c)$, of events with $\Delta c$ characters entered between catastrophic cascades, i.e. cascades which reached line 1000. Circles: Number distribution, $N\left(c_{1000}\right)$, of events with total length of random walks, $c_{1000}$, for catastrophic cascades. The inset is a magnified view of the region around the origin. The parameters of this simulation are identical to the ones used for Fig. 1(a).

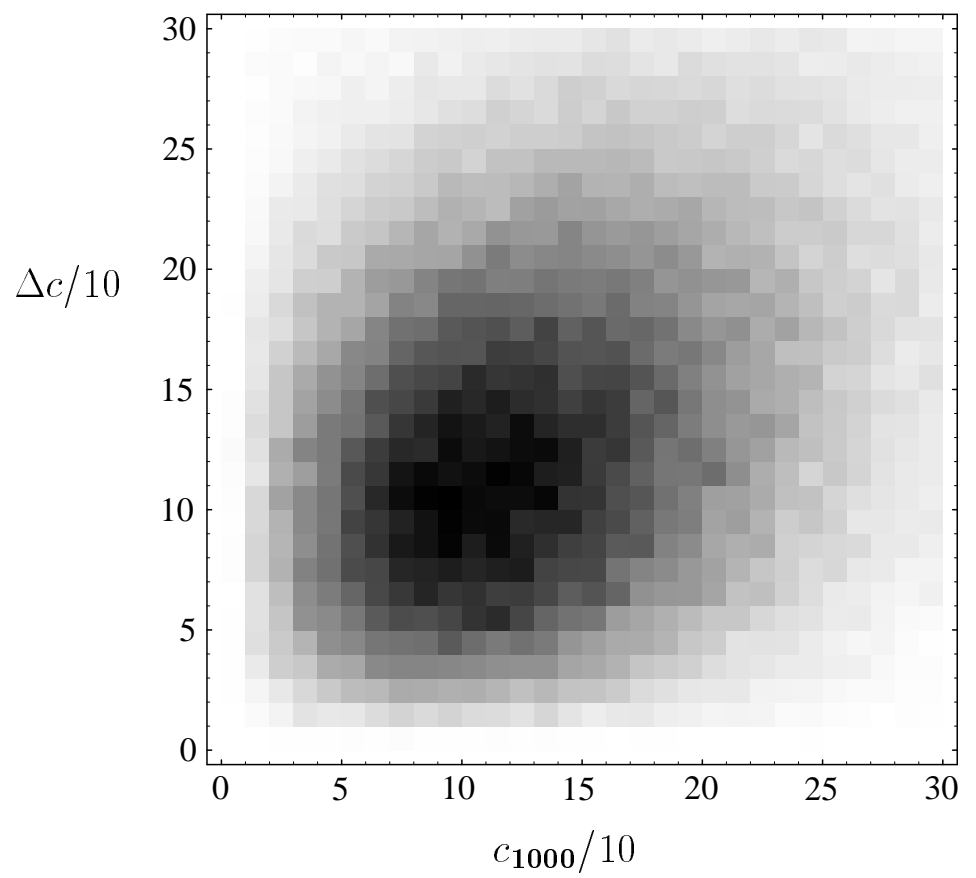

FIG. 3. Contour plot of the number distribution, $N\left(c_{1000}, \Delta c\right)$, of catastrophic cascades with random walks of total length $c_{1000}$ and number of characters entered, $\Delta c$, before the next catastrophic cascade. The grey level is proportional to $N\left(c_{1000}, \Delta c\right)$, with black representing the maximum and white a value of 0 .

Since $\Delta c$ represents the number of characters entered between catastrophic cascades, and $c_{1000}$ is the number of characters removed by a catastrophic cascade, sum rules require that $N(\Delta c)$ and $N\left(c_{1000}\right)$ have the same norm and mean. The surprising aspect of Fig. 2 is that both distributions are identical, even down to the quadratic rise near 
the origin (see insert). The connection with the diffusion equation explains the Wigner-distribution form of $N\left(c_{1000}\right)$, but not for $N(\Delta c)$.

Despite the same functional shape, the above two distributions are not tightly correlated. Fig. 3 shows a density plot of the number distribution $N\left(c_{1000}, \Delta c\right)$, where the grey level is proportional to the number of counts in a given bin. One sees only a weak enhancement of this number distribution along the diagonal. Here we plot the correlation between $c_{1000}$ and the time delay to the next catastrophic cascade, but we obtain virtually identical results when plotting the correlation between $c_{1000}$ and the time delay since the previous catastrophic cascade.

The total number of trailing blanks summed over all lines up to a certain maximum (here: 1000) changes by -1 each time a new character is entered. Catastrophic cascades change the total number of blanks by $c_{1000}$. Thus the total number of trailing blanks has exactly identical time dependence as (up to a minus sign) the total mass of the sandpile measured in ref. [9].

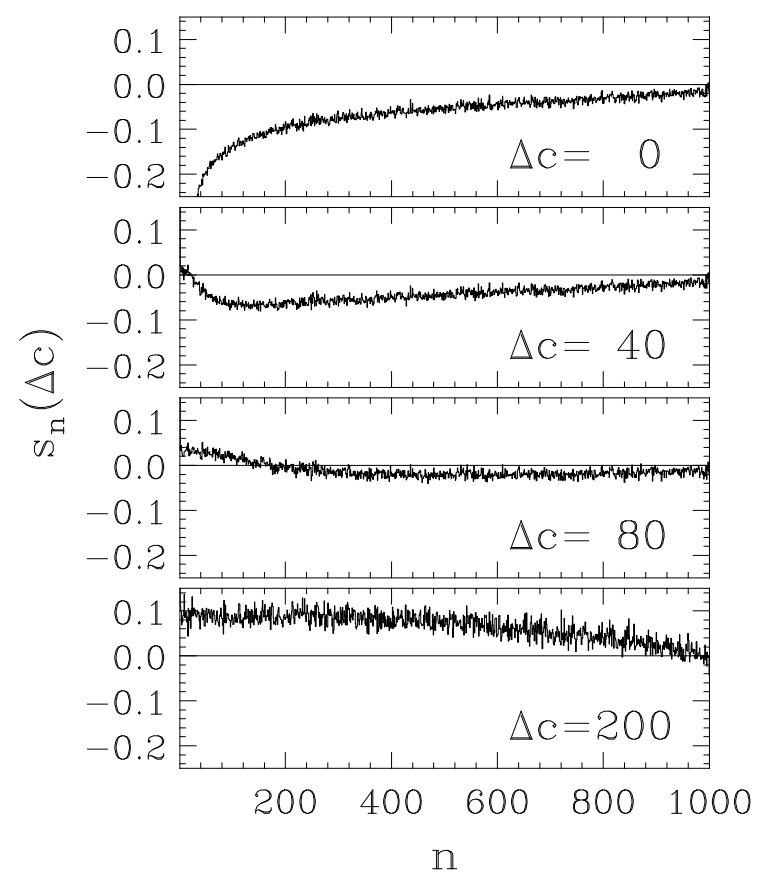

FIG. 4. Average stress, $s_{n}(\Delta c)$, as a function of the line number, $n$, and the number of characters entered since the last catastrophic cascade, $\Delta c$.

In order to understand the trends of the buildup and release we compute the average stress in each line,

$$
s_{n}(\Delta c)=\left\{\left\langle b_{n}\right\rangle-b_{n}\right\}_{\Delta c},
$$

as a function of the time delay since the last catastrophe. (Here, the notation $\{\ldots\}_{\Delta c}$ indicates averaging over all events with identical value of $\Delta c$.) This is done in Fig. 1 . We see from our data in Fig. 1 that a catastrophic cascade typically inserts more extra blanks in the early lines, thus reducing the stress in them below the average level. This indicates that large positive steps early in the random walk are correlated with catastrophic events. However, the structure of the stress as a function of the line number demonstrates the complex nature of the evolution of the self-organized critical state. This behavior cannot be explained in terms of a simple random walk.

In conclusion, we have examined self-organized criticality in line-wrap cascades in word processors. We find that the distribution of cascade lengths and cascade strengths can be accurately modeled with the diffusion equation and compared to analytic forms. We find that the issue of predictability is complex. Although the distribution of times between large cascades is of a simple Wigner-distribution form, stress develops in a rather complicated style. Even though the present system represents the simplest non-trivial example of self ordered criticality, the model inspires a wealth of questions, several of which we have addressed analytically and many more which remain unresolved such as what is the optimum way to predict the onset of large cascades. By conquering this easily-modeled example, insight 
may be reached regarding more complex systems such as sand piles or more pertinent problems such as the modeling of earth quakes.

It is also possible to extend the model to higher dimensionality or to incorporate the addition of stress all through the paragraph rather than only through the first line. Work in these directions is currently in progress.

This research was supported by NSF grant PHY-9403666, and by an NSF Presidential Faculty Fellow award (W.B.), NSF grant PHY-9253505.

[1] P. Bak, C. Tang, and K. Wiesenfeld, Phys. Rev. Lett. 59, 381 (1987).

[2] P. Bak and K. Chen, Scientific American, January 1991, p. 46.

[3] P. Bak and M. Creutz, in Fractals in Science, ed. A. Bunde and S. Havlin (Springer, Heidelberg, 1994 ), p. 26.

[4] S.K. Grumbacher, K.M. McEwen, D.A. Halvorsen, D.T. Jacobs, and J. Lindler, Am. J. Phys. 61, 329 (1993).

[5] B. Gutenberg and C.F. Richter, Ann. Geofis. 9, 1 (1956).

[6] J.M. Carlson and J.S. Langer, Phys. Rev. Lett. 62, 2632 (1989).

[7] D. Dhar and R. Ramaswamy, Phys. Rev. Lett. 63, 1659, (1989).

[8] D. Dhar, Phys. Rev. Lett. 64, 1613 (1990).

[9] J. Rosendahl, M. Vekić, and J.E. Rutledge, Phys. Rev. Lett. 73, 537 (1994).

[10] S. Field, J. Witt, and F. Nori, Phys. Rev. Lett. 74, 1206 (1995). 